\title{
Virtual Sensor Based on a Deep Learning Approach for Estimating Efficiency in Chillers ${ }^{\star}$
}

\author{
Serafín Alonso 1[0000-0003-3467-4938], Antonio Morán ${ }^{10000-0002-2762-6949]}$, \\ Daniel Pérez ${ }^{1[0000-0002-3921-1599]}$, Perfecto Reguera ${ }^{10000-0002-3346-5109]}$, \\ Ignacio Díaz ${ }^{2[0000-0003-0420-2315]}$, and Manuel Domínguez ${ }^{10000-0002-3921-1599]}$ \\ 1 Grupo de investigación en Supervisión, Control y Automatización de Procesos \\ Industriales (SUPPRESS), Esc. de Ing. Industrial e Informática, Universidad de \\ León, Campus de Vegazana s/n, 24007, León, Spain. http://suppress.unileon.es \\ \{saloc, a.moran, dperl, prega, manuel.dominguez\}@unileon.es \\ 2 Electrical Engineering Department, University of Oviedo, Edif. Departmental 2, \\ Campus de Viesques s/n, Gijón 33204 Spain \\ idiaz@uniovi.es
}

\begin{abstract}
Intensive use of heating, ventilation and air conditioning (HVAC) systems in buildings entails an analysis and monitoring of their efficiency. Cooling systems are key facilities in large buildings, and particularly critical in hospitals, where chilled water production is needed as an auxiliary resource for a large number of devices. A chiller plant is often composed of several HVAC units running at the same time, being impossible to assess the individual cooling production and efficiency, since a sensor is seldom installed due to the high cost. We propose a virtual sensor that provides an estimation of the cooling production, based on a deep learning architecture that features a 2D CNN (Convolutional Neural Network) to capture relevant features on two-way matrix arrangements of chiller data involving thermodynamic variables and the refrigeration circuits of the chiller unit. Our approach has been tested on an air-cooled chiller in the chiller plant at a hospital, and compared to other state-of-the-art methods using 10-fold cross-validation. Our results report the lowest errors among the tested methods and include a comparison of the true and estimated cooling production and efficiency for a period of several days.
\end{abstract}

Keywords: HVAC systems · Efficiency · Cooling power · Virtual Sensor · Deep Learning · Convolutional Neural Network.

\footnotetext{
* This work was supported in part by the Spanish Ministerio de Ciencia e Innovacion (MICINN) and the European FEDER funds under project CICYT DPI2015-69891$\mathrm{C} 2-1-\mathrm{R} / 2-\mathrm{R}$.

This is an author-created version of a contribution published in Communications in Computer and Information Science. The final publication is available at Springer via http://dx.doi.org/10.1007/978-3-030-20257-6_26
} 


\section{Introduction}

Energy consumption in large buildings, represents more than $20 \%$ of the global energy consumption in developed countries. The proliferation of heating, ventilation and air conditioning (HVAC) systems is one of the main reasons behind such a high consumption [16]. In central air conditioning systems, chillers are the main energy consumers, consuming more than $40 \%$ of the total energy in commercial and industrial buildings [17. Thus, their efficiencies have a significant effect on the overall energy performance of these buildings.

Several Energy Efficiency Indicators (EEI) can be used to determine the chiller efficiency [1. Most of the EEI require measuring the cooling power delivered to chilled water (the chiller output). However, manufacturers do not usually include energy meters in their chiller designs due to high installation, maintenance and recalibration costs [14. A physical cooling meter can be replaced by a virtual sensor [13].

Virtual sensors refer to software, usually including mathematical models that allow measuring process variables or quantities using indirect measurements of related variables, useful in cases where physical sensors are not available, expensive, slow or imprecise. They are a low-cost and non-invasive choice to obtain observations from a real system [15] and have been widely applied for flow and efficiency metering in cooling plants [19]18. Data based models for virtual sensors using radial basis functions, multilayer perceptrons and other machine learning methods, can provide accurate estimations for cooling power [11]3] provided input-output training data are available, which in cooling systems can be acquired with portable energy meters.

Recent deep learning methods have been used for time ahead cooling prediction 446. However, those methods have been barely applied for estimating the current output of a virtual sensor. Therefore, we suggest that deep learning methods can achieve more accurate models for virtual sensors of cooling power. We propose here a virtual sensor based on a deep learning approach for estimating the cooling production and efficiency in chillers. The proposed model, based on 2D Convolutional Neural Network (2D CNN) [12, is compared to other state-of-the-art methods and tested on a real air-cooled chiller of the plant at the Hospital of León achieving better results.

This paper is organized as follows: Section 2 states the problem. In Section 3. the adopted methodology is exposed. Here, the proposed deep approach is explained in detail. In Section 4, the experiment is presented and results are discussed. Finally, conclusions and future work are drawn in Section 5.

\section{Problem Statement}

\subsection{Chiller efficiency}

A chiller is an HVAC system in charge of providing cooling energy to building facilities. Normally, a chiller is formed by a set of refrigeration circuits with similar or even different capacity in order to achieve a better adaptation to 


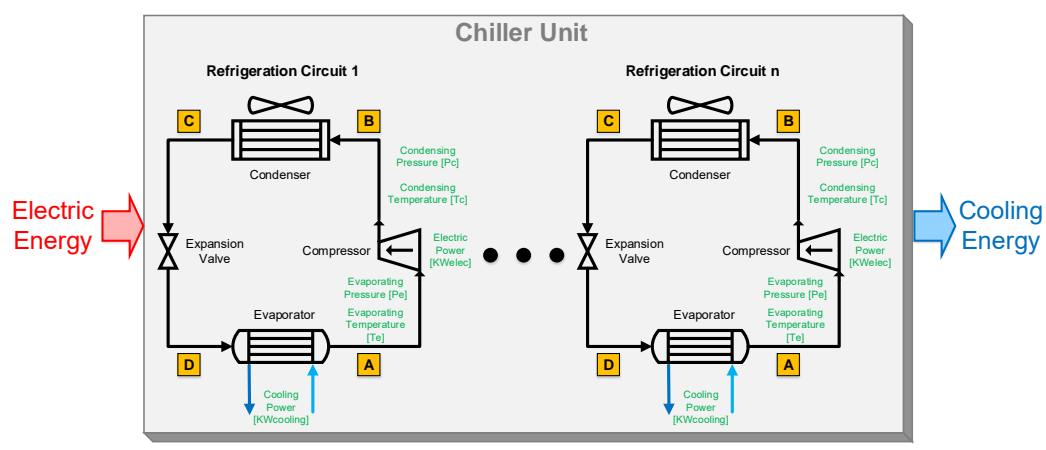

Fig. 1. A chiller unit with several refrigeration circuits.

variable cooling loads (see Fig. 1). Each individual circuit provides cooling power according to central chiller control. The total cooling production of the chiller is the sum of the cooling power from each circuit.

Measuring the total cooling production of a chiller is crucial to know the chiller efficiency over time. Typically, chiller manufacturers use the COP (coefficient of performance) indicator as an efficiency ratio, providing theoretical values for given surrounding conditions during the manufacturing process. However, chiller efficiency varies over time and depends on external conditions, so it is required to monitor this performance indicator. COP values can be easily computed using the total cooling power and electric power (see Eq. 1).

$$
C O P=\frac{\text { CoolingPower }[K W]}{\text { ElectricPower }[K W]}=\frac{K W \text { cooling }}{K W \text { elec }}=\frac{\sum_{i=1}^{n} K W \text { cooling }_{i}}{\sum_{i=1}^{n} K W \text { elec }_{i}}
$$

Manufacturers incorporate electric power meters in the chillers since they use the compressor current for controlling cooling capacity. However, they seldom include cooling power meters, because it is not essential for capacity control and increases the cost of the chiller. Moreover, cooling power meters are based on a flow meter, whose measuring principle is usually invasive, thereby becoming a new drawback for their installation. Furthermore, chiller units used to be placed outside so, low temperatures could damage the flow meter. Therefore, virtual sensor implementation becomes crucial in order to measure the cooling power and to compute COP value in a chiller. The virtual sensor should be able to estimate the cooling production and the COP value based on internal variables of the refrigeration circuits.

Applying the energy conservation equation $Q-W=\Delta H$ to the theoretical refrigeration cycle [10, we have $Q_{\text {evaporator }}=\Delta H=H_{D}-H_{A}$. So, cooling production can be obtained using Eq. 2 .

$$
K W_{\text {cooling }}=Q_{\text {evaporator }} \varepsilon=\left(H_{D}-H_{A}\right) \varepsilon
$$

$\varepsilon$ is the efficiency of the heat exchanger. It demonstrates that cooling production depends mainly on enthalpies $H$ in the evaporator input and output (see Fig. 
11. On the other hand, COP value of the theoretical refrigeration cycle can be defined as follows (see Eq. 3):

$$
C O P=\frac{Q_{\text {evaporator }}}{W_{\text {compressor }}}=\frac{H_{D}-H_{A}}{H_{A}-H_{B}}
$$

It proves that chiller performance (COP) depends mainly on enthalpies $H$. The enthalpy of an ideal gas provides information about internal energy variations and depends mainly on kinetic and vibration energies of molecules, i.e., on temperature and pressure. On the other hand, the enthalpy is a magnitude which characterizes the state of a system in equilibrium, but it does not consider how the system reaches that state. Thus, it can be stated that cooling production and COP depend on enthalpies (given the type of refrigeration gas), i.e, gas pressure and temperature in suction and discharge lines and compressor work. Our hypothesis is that these variables can define the state of the system.

\subsection{Air-cooled chiller at the Hospital of León}

The chiller plant at the Hospital of León has been used in the experimental setup. Basically, that plant consists of 5 air-cooled and 2 water-cooled chillers. An air-cooled chiller is used for the experiments.

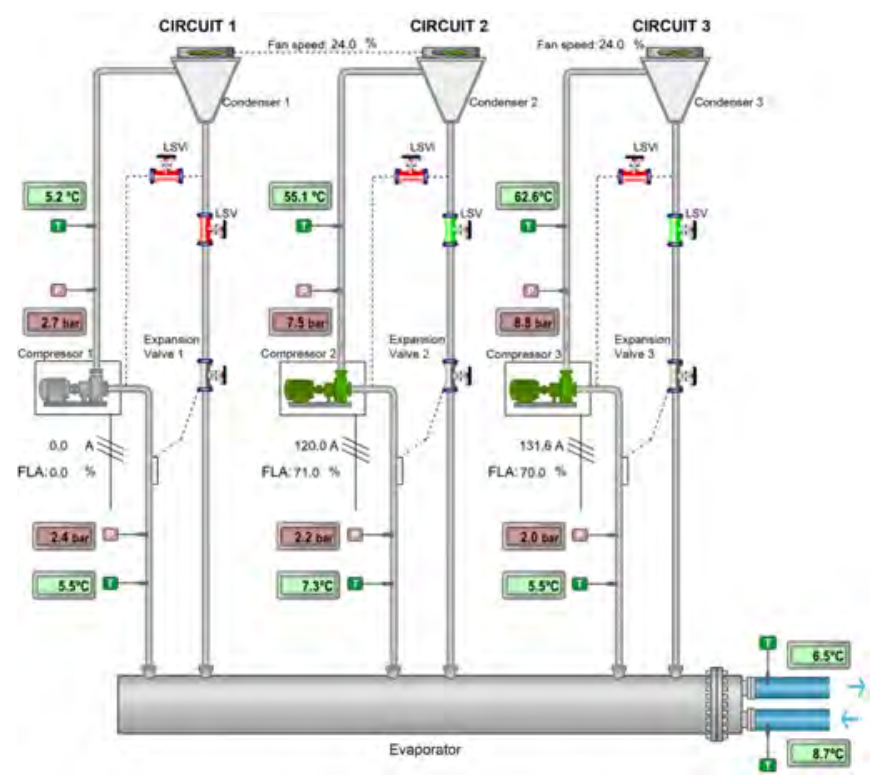

Fig. 2. The air-cooled chiller with 3 refrigeration circuits.

The air-cooled chiller (model Petra APSa 400-3) has a maximum cooling capacity of 400 tons (approximately $1407 \mathrm{~kW}$ ) and includes 3 refrigeration circuits 
Table 1. Main internal variables for each refrigeration circuit of the chiller.

\begin{tabular}{|c|c|c|}
\hline Symbol & Name & Unit \\
\hline $\mathrm{Te}$ & Evaporating temperature & ${ }^{\circ} C$ \\
$\mathrm{Pe}$ & Evaporating pressure & $\mathrm{KPa}$ \\
$\mathrm{Tc}$ & Condensing temperature & ${ }^{\circ} C$ \\
$\mathrm{Pc}$ & Condensing pressure & $\mathrm{KPa}$ \\
KWelec & Compressor electric power & $\mathrm{KW}$ \\
KW cooling & Cooling power & $\mathrm{KW}$ \\
\hline
\end{tabular}

(see Fig. 2). Each one is composed of a screw compressor, an electronic expansion valve (EEV) and a condenser in $\mathrm{V}$ form. A common evaporator is used for the 3 circuits. The compressor, driven by a three-phase induction motor $(400 \mathrm{~V} ; 109$ $\mathrm{kW}$ ), has a maximum displacement of $791 \mathrm{~m}^{3} / \mathrm{h}$ of R134a refrigeration gas. Its capacity can be regulated between 50-100 \% of maximum value by means of two auxiliary load and unload valves. The condensers have 16 fans of $1.5 \mathrm{~kW}$, driven by variable speed drives. Note that the condensing control signal is common to the 3 circuits. The control board regulates the operation of 3 refrigeration circuits. It communicates with a BMS (Building Management System) which collects and stores data from main internal variables (listed in Table 1) using Modbus protocol.

It should be remarked that it is impossible to measure the individual cooling production of each refrigeration circuit, since the evaporator is common to all the circuits and only the total cooling production is accessible for measuring. Moreover, the condensing control signal is common for the 3 refrigeration circuits, so overpressures in one circuit can affect to the other two circuits (assuming all of them are running). Thus, some interactions among the refrigeration circuits are expected in this chiller. On the other hand, dependencies among variables are expected since the refrigeration cycle is closed, i.e, suction and compressor variables will determine the evolution of discharge variables.

\section{Methodology}

Based on the considerations exposed in section 2, the proposed approach should take into account the following aspects in order to address the regression problem:

- A chiller unit can comprise several refrigeration circuits, which provide cooling energy.

- Cooling energy and efficiency depend on the state of the refrigeration circuits, which is defined by internal variables (pressures, temperatures and compressor work).

- Interactions among refrigeration circuits could appear, depending on the chiller manufacturing structure.

- Dependencies among variables are expected since the refrigeration cycle is closed. 
The cooling production of a refrigeration circuit can be defined as a function $f$ of its main internal variables (see $\mathrm{Eq} 4$ ).

$$
K W \text { cooling }_{i}=f\left(T e_{i}, P e_{i}, T c_{i}, P c_{i}, K W e l e c_{i}\right) \quad \forall i \in\{1, \ldots, n\}
$$

According to this, a virtual sensor for the overall chiller production ( $K W$ cooling) can be designed using the next regressor

$$
\begin{aligned}
& T e_{1}, P e_{1}, T c_{1}, P c_{1}, K W e l e c_{1}, T e_{2}, P e_{2}, T c_{2}, P c_{2}, K W e l e c_{2}, \ldots \\
& \ldots, \ldots, \ldots, \ldots, \ldots, \ldots, \ldots, \ldots, T e_{n}, P e_{n}, T c_{n}, P c_{n}, K W e l e c_{n}
\end{aligned}
$$

and obtaining a model function $f$ that relates it to $K W$ cooling. To model $f$, we propose treating the instances of regressor (5) as images of size (circuits $\times$ variables), which define the state of the chiller. Then, we propose to use a $2 \mathrm{D}$ convolutional layer to allow us to extract relationships among refrigeration circuits and variables. As proved in Section 2, the chiller state can be characterized by internal variables of each refrigeration circuit. Let us suppose several "photos" of the chiller are taken over time. These images contain information about different chiller states that can be used as input data. This suggests the use of an image processing method which allows to detect patterns in 2D images. Our approach is based on a 2D Convolutional Neural Network (2D CNN) which takes advantage of coherence among refrigeration circuits and variables of the chiller.

The adopted methodology is depicted in Fig. 3. First, real data are collected from chiller. Data from internal variables (see Table 1) and from cooling power are required. These data are merged and preprocessed. Next, the deep approach is trained and validated in order to choose the best model function $f$ for the virtual sensor and to delimit its error range. Finally, the model is deployed as a virtual sensor, enabling monitoring and providing the estimation of cooling power and COP computation. Note that COP is computed from the estimated cooling power and the measured electric power.

\subsection{Deep learning model architecture}

Our approach is based on 2D Convolutional Neural Network (2D CNN). It consists of several layers: an input layer whose dimension is (circuits $\times$ variables), a 2D CNN layer to detect relationships among circuits and variables, a fully connected layer and an output layer with dimension 1 . The choice of the filter kernel is crucial since it should consider all possible pair-wise combinations among circuits and also among variables, so it should be $(2,2)$. If the number of circuits is too high, data augmentation can be required to obtain new images by shuffling circuits. Note that, the number of pair-wise relationships among circuits is given by $\frac{c^{2}-c}{2}$, assuming a filter kernel of $(2,2)$.

A downsampling layer is not required since the size of the images is expected to be small. A nonlinear activation function is used for all units. Then, a fully

connected layer is applied with resulting units. Finally, an output layer with dimension 1 provides cooling estimation as a virtual sensor measurement. 


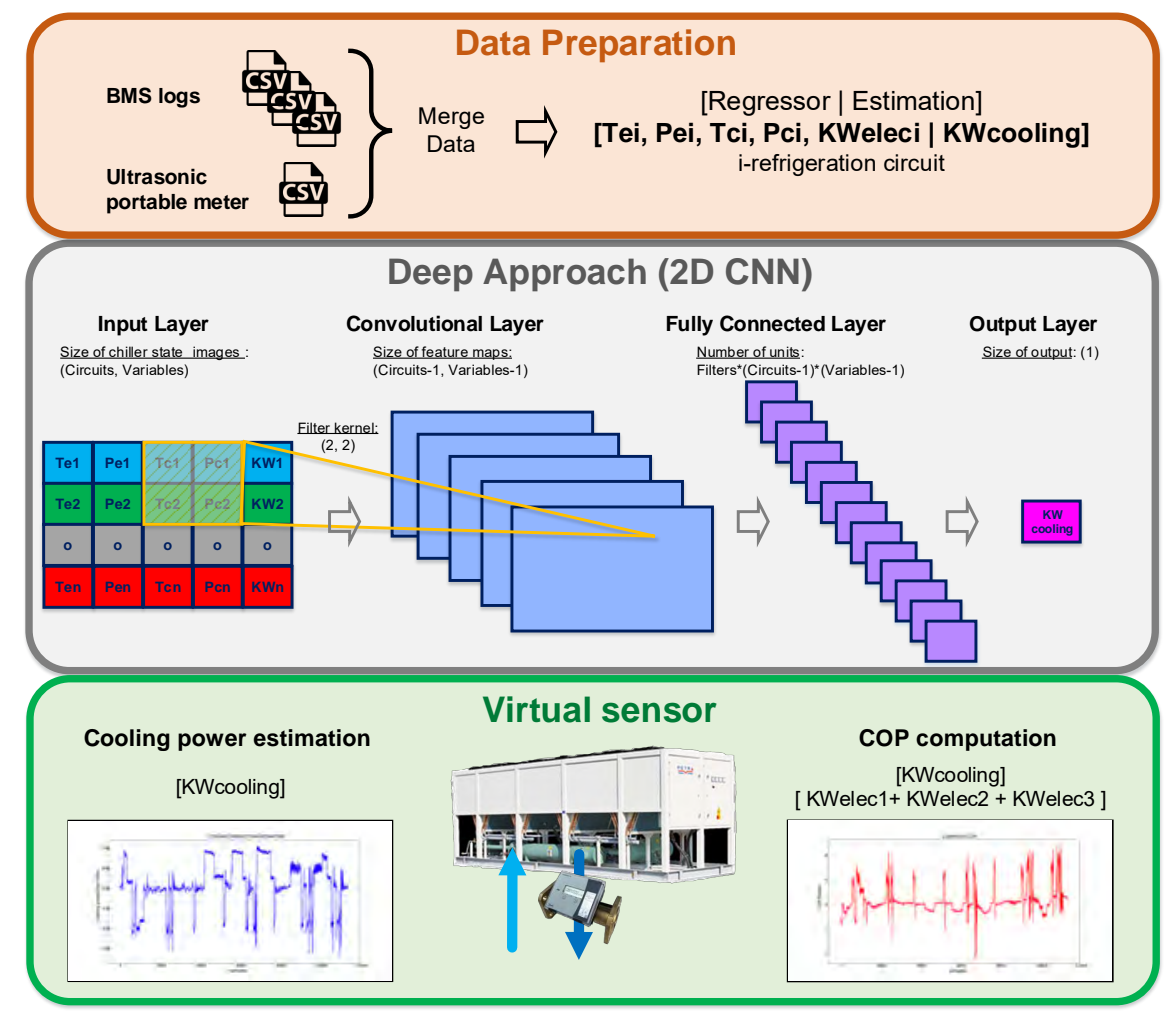

Fig. 3. Methodology based on a Deep Learning approach.

The proposed deep approach is compared with other linear and non-linear methods used widely in the literature: a) two linear methods, including Multiple Linear Regression (MLR) [8] and Random Sample Consensus (RANSAC), based on selecting uniformly at random a subset of data samples to estimate model parameters [5]; and b) several nonlinear methods, including a kernel method Support Vector Regression (SVR) [2], a shallow Multilayer Perceptron (Shallow MLP), with just one hidden layer [9] and trained using backpropagation algorithm, a deep Multilayer Perceptron (Deep MLP) with many hidden layers using a special initialization strategy to avoid the vanishing/exploding gradient problem [7.

\section{Results}

\subsection{Collecting Data}

Data have been collected from two sources. First, we have gathered data from BMS (Building Management System) logs (plant manager subsystem). BMS 
stores these data when changing in order to optimize storage capacity. The second data source is an ultrasonic portable meter (Fluxus F601 by Flexim). It supplies the lack of cooling power meter in the chiller. Cooling power is acquired and stored each minute from flow and leaving and return chilled water temperatures. Both data sources (CSV format) were preprocessed. For that, data from BMS logs were resampled with 1 minute and then synchronized and merged with data from ultrasonic portable meter.

\subsection{Experiments}

An experiment has been performed to test our approach. Data from air-cooled chiller no. 5 were selected from 2 months (2018 December and 2019 January), with a sampling time of 1 minute, so the number of samples was 38169. Note that, that chiller was not running consecutive time since its operation was alternated with other chillers in the plant. Five regressor variables are used, $T_{e}, P_{e}, T_{c}, P_{c}, K W$ elec (see Table 1) for each of the 3 refrigeration circuits, resulting in 15 regressor variables. The estimated variable was the cooling power $K W$ cooling. Total data were split into 2 datasets: The training and test model dataset (70\% of total data) is used to train and test all models. The proposed approach, 2D CNN, and the remaining linear and non-linear models are trained using this dataset. A 10-fold cross validation has been applied to test models and select the best one. The virtual sensing test dataset (30\% of total data) is used to test virtual sensor estimation of cooling power and COP. In this case, we suppose that the F601 portable meter is disconnected and the virtual sensor based on the proposed 2D CNN model is used to measure cooling production and efficiency.

The hyperparameters for each model were tuned after several preliminary experiments, choosing the best ones in each scenario. The proposed $2 \mathrm{D}$ CNN model consists of a 4 layers, an input layer $(3,5,1)$, a 2D CNN layer $(2,4$, 32 ), a flatten layer (256) and a dense output layer (1). A dropout regularization (0.001) was applied to avoid overfitting. Activation relu function was selected. The number of filters was 32 with a filter kernel of $(2,2)$ in order to detect pairwise patterns among circuits and variables. The padding was defined as valid, achieving feature maps with a lower dimension. No downsampling is required due to small size of input images.

The SVR model uses a radial basics function as kernel with 0.01 gamma coefficient. The penalty parameter $\mathrm{C}$ of the error was established in 0.001 and the epsilon-tube within which no penalty is associated in the training loss function was 0.01 .

The Shallow MLP model consists of 3 layers, a input layer with a dimension of 15 , a hidden layer (64 units) and an output layer (1 unit). It is trained with backpropagation algorithm. The Deep MLP model consists of 12 layers, a input layer with a dimension of 15, 10 hidden identical layers (64 units) and an output layer (1 unit). The dropout regularization was 0.001 to avoid overfitting and relu was selected as activation function (also for Shallow MLP). For all methods, the training epochs were 1000 . 


\subsection{Model validation}

A 10-fold cross validation has been carried out to test and validate our approach. Using training and test dataset (70\% of total samples), 2D CNN and the remaining methods have been trained and tested. MAE (Mean Absolute Error), MAPE (Mean Absolute Percentage Error) errors have been selected as evaluation metrics since that values are easily understood by any engineer. Average errors and standard deviations of each 10-fold iteration have been computed. Two additional evaluation metrics are also included in order to compare the performance of our approach (and that of the other methods) against the linear (MLR) approximation, which provides a closed-form and fast solution and so, it is taken as a baseline reference:

$$
M A E_{R}=1-\frac{M A E_{\left(\text {method }_{m}\right)}}{M A E_{(M L R)}} ; \quad M A P E_{R}=1-\frac{M A P E_{\left(\text {method }_{m}\right)}}{M A P E_{(M L R)}}
$$

They allow us to check how much the scores of a certain method improve $(+$ errors) or worsen (- errors) with respect to MLR scores (reference method).

According to train errors (see Table 2), Deep MLP is the best method, improving around $67 \%$ a MLR and nearly $13 \%$ the second one (Shallow MLP). Our approach is the third method providing also very low errors (MAPE is $1.85 \%$ and MAE is $8.96 \mathrm{KW}$ ).

According to test errors (see Table 2 and focusing on MAPE errors, our approach is the best method, improving around $29 \%$ a MLR and nearly $13 \%$ the second one (SVR). The difference with the following methods (Deep MLP and Shallow MLP) is $14 \%$ and $18 \%$, respectively. Checking MAE errors, our approach is also the winner. It can be stated we could estimate cooling production and efficiency either with a relative error of $3.04 \%$ or with an absolute error of 13.24 KW (see Fig. 4). Considering the standard deviation $( \pm 1.23)$, relative errors can range between $1.81 \%$ and $4.27 \%$ (in the best and the worst scenario). Therefore, we can conclude that $2 \mathrm{D} \mathrm{CNN}$ is the best method to build the virtual sensor.

\subsection{Virtual sensor test and COP computation}

Once the 2D CNN model was validated, we performed a new test using virtual sensing dataset (30\% of the total data). Now, we suppose the portable F601

Table 2. Training and test errors.

\begin{tabular}{|c||c|c|c|c||c|c|c|c|}
\hline \multicolumn{1}{|c||}{} & \multicolumn{4}{c||}{ Training Dataset } & \multicolumn{3}{c|}{ Test Dataset } \\
\hline Method & $\begin{array}{c}\text { MAE } \\
(\text { mean } \pm \text { std })\end{array}$ & $M A E_{R}$ & $\begin{array}{c}\text { MAPE } \\
(\text { mean } \pm \text { std })\end{array}$ & $M A P E_{R}$ & $\begin{array}{c}\text { MAE } \\
\text { (mean } \pm \text { std })\end{array}$ & $M A E_{R}$ & $\begin{array}{c}\text { MAPE } \\
(\text { mean } \pm \text { std })\end{array}$ & $M A P E_{R}$ \\
\hline MLR & $16.64 \pm 0.65$ & 0 & $3.55 \pm 0.15$ & 0 & $19.80 \pm 5.94$ & 0 & $4.27 \pm 1.62$ & 0 \\
RANSAC & $18.25 \pm 2.70$ & -0.10 & $4.31 \pm 0.89$ & -0.21 & $20.43 \pm 5.80$ & -0.03 & $4.76 \pm 1.75$ & -0.11 \\
SVR & $10.23 \pm 0.34$ & 0.38 & $2.14 \pm 0.09$ & 0.40 & $15.82 \pm 6.89$ & 0.20 & $3.58 \pm 1.88$ & 0.16 \\
Shallow MLP & $7.94 \pm 0.31$ & 0.52 & $1.65 \pm 0.08$ & 0.54 & $15.95 \pm 6.58$ & 0.19 & $3.79 \pm 2.19$ & 0.11 \\
Deep MLP & $\mathbf{5 . 5 3} \pm \mathbf{0 . 4 0}$ & $\mathbf{0 . 6 8}$ & $\mathbf{1 . 1 3} \pm \mathbf{0 . 0 9}$ & $\mathbf{0 . 6 7}$ & $16.66 \pm 6.85$ & 0.16 & $3.64 \pm 1.58$ & 0.15 \\
2D CNN & $8.96 \pm 0.46$ & 0.46 & $1.85 \pm 0.09$ & 0.48 & $\mathbf{1 3 . 2 4} \pm \mathbf{3 . 7 1}$ & $\mathbf{0 . 3 3}$ & $\mathbf{3 . 0 4} \pm \mathbf{1 . 2 3}$ & $\mathbf{0 . 2 9}$ \\
\hline
\end{tabular}




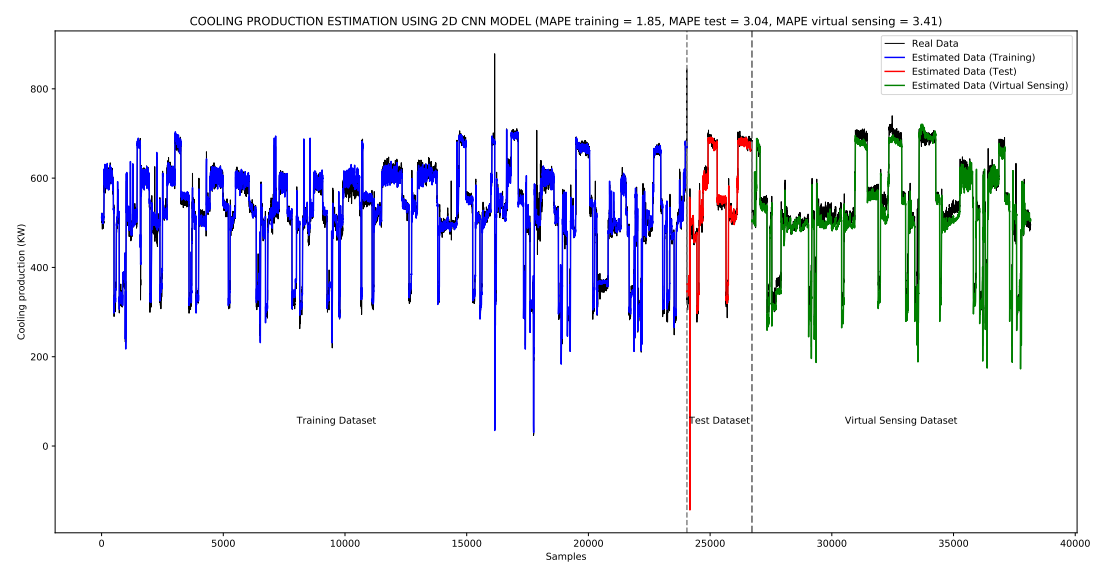

Fig. 4. Cooling production estimation using the proposed deep approach (2D CNN) for training, test and virtual sensing datasets.

meter is disconnected from the chiller and we try to verify the virtual sensor measurement, estimating the cooling production and efficiency. In this case, we have chosen MAPE error to test estimations.

The results can be observed in Fig. 5 . The virtual sensor provides measurements of cooling power with an error of $3.41 \%$ and with an absolute error of 16.43 KW. It is very accurate, except when starting or stopping a compressor.

The final aim of virtual sensor is to monitor chiller efficiency. For that, COP value is computed using estimated cooling power and measured electric power (sum of 3 electric compressor powers) for all virtual sensing dataset. The result can be seen in Fig. 6. The chiller efficiency can be estimated with a relative error of $3.41 \%$ and with an absolute error of 0.16 . Note that the maximum errors occur when a compressor starts or stops.

\section{Conclusions}

In this paper, we have proposed a virtual sensor for cooling power estimation based on available internal chiller variables (temperatures, pressures and compressor power) and using a deep convolutional neural network (2D CNN). The proposed architecture uses a convolutional layer that takes advantage of coherence between the three refrigeration circuits of the chiller, and was systematically compared to a set of state-of-the-art methods, using several performance metrics with a 10 -fold validation methodology, where our proposed method achieved the best results.

On the application side, the developed virtual sensor is very valuable for several reasons. First, the estimations of the virtual sensor can replace the current measurements from the expensive portable measuring system used for training, that can be only available provisionally. This methodology can be extensible 


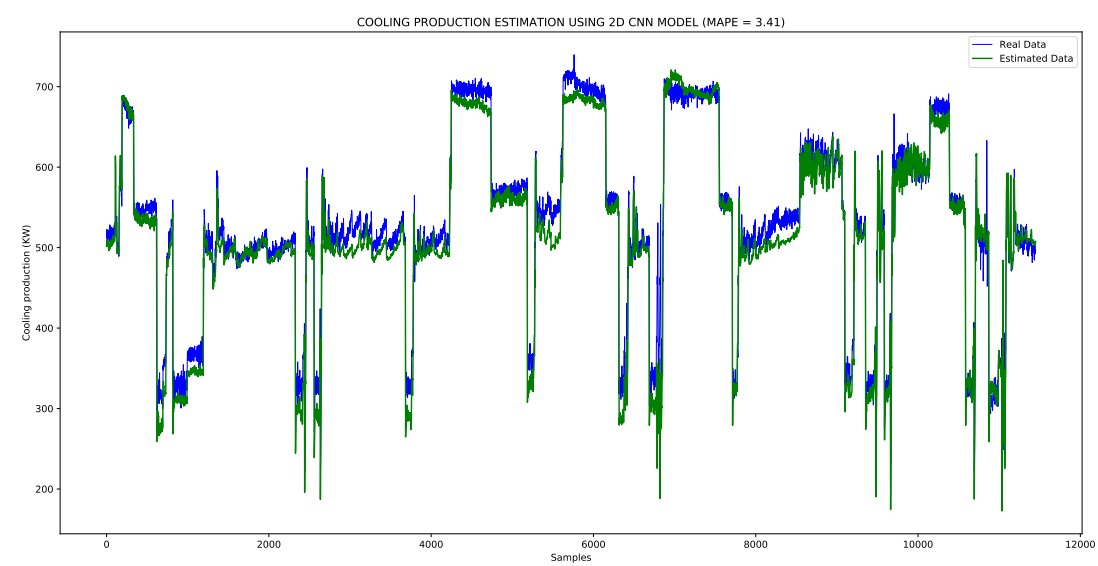

Fig. 5. Cooling production estimation using for virtual sensing dataset.

("copy-pasted") to any chiller, specially to the 5 identical chillers in this plant, resulting in a highly cost-effective way to track and monitor the overall cooling power of the plant. Second, the availability of electric power consumption and an accurate enough estimation of cooling power allows to have also an estimation of the chiller efficiency, highly valuable for energy optimization of the overall plant.

\section{References}

1. Alves, O., Monteiro, E., Brito, P., Romano, P.: Measurement and classification of energy efficiency in HVAC systems. Energy and Buildings 130, 408 - 419 (2016). https://doi.org/10.1016/j.enbuild.2016.08.070

2. Cortes, C., Vapnik, V.: Support-vector networks. Machine Learning 20(3), 273-297 (1995), www.scopus.com cited By (since 1996): 4606

3. Escobedo-Trujillo, B., Colorado, D., Rivera, W., Alaffita-Hernández, F.: Neural network and polynomial model to improve the coefficient of performance prediction for solar intermittent refrigeration system. Solar Energy 129, 28 - 37 (2016). https://doi.org/10.1016/j.solener.2016.01.041

4. Fan, C., Xiao, F., Zhao, Y.: A short-term building cooling load prediction method using deep learning algorithms. Applied Energy 195, 222 - 233 (2017). https://doi.org/10.1016/j.apenergy.2017.03.064

5. Fischler, M.A., Bolles, R.C.: Random sample consensus: a paradigm for model fitting with applications to image analysis and automated cartography. Communications of the ACM 24(6), 381-395 (1981)

6. Fu, G.: Deep belief network based ensemble approach for cooling load forecasting of air-conditioning system. Energy 148, 269 - 282 (2018). https://doi.org/10.1016/j.energy.2018.01.180

7. Goodfellow, I., Bengio, Y., Courville, A.: Deep Learning. MIT Press (2016), http: //www.deeplearningbook.org

8. Hastie, T., Tibshirani, R., Friedman, J.: The elements of statistical learning. Second Edition. Springer (2009) 


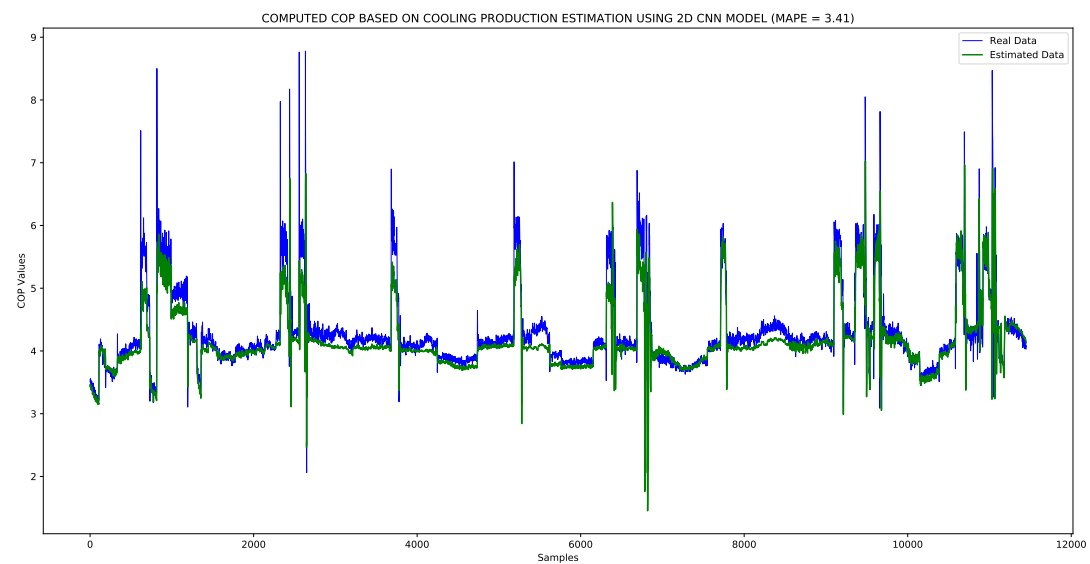

Fig. 6. Computed COP values using cooling production estimation.

9. Haykin, S.S.: Neural networks and learning machines. 3rd Edition. Pearson Education (2009)

10. Klein, S.A.: Design considerations for refrigeration cycles. In: International Refrigeration and Air Conditioning Conference. vol. 190, pp. 511 - 519. Purdue e-Pubs (1992), http://docs.lib.purdue.edu/iracc/190

11. Kusiak, A., Li, M., Zheng, H.: Virtual models of indoor-air-quality sensors. Applied Energy 87(6), 2087 - 2094 (2010). https://doi.org/10.1016/j.apenergy.2009.12.008

12. LeCun, Y., Bengio, Y.: The handbook of brain theory and neural networks. chap. Convolutional Networks for Images, Speech, and Time Series, pp. 255-258. MIT Press, Cambridge, MA, USA (1998), http://dl.acm.org/citation.cfm?id= 303568.303704

13. Li, H., Yu, D., Braun, J.E.: A review of virtual sensing technology and application in building systems. HVAC\&R Research 17(5), 619-645 (2011). https://doi.org/10.1080/10789669.2011.573051

14. Mcdonald, E., Zmeureanu, R.: Virtual flow meter to estimate the water flow rates in chillers. ASHRAE Transactions 120, 200-208 (06 2014)

15. McDonald, E., Zmeureanu, R.: Development and testing of a virtual flow meter tool to monitor the performance of cooling plants. Energy Procedia 78, 1129 1134 (2015). https://doi.org/10.1016/j.egypro.2015.11.071

16. Pérez-Lombard, L., Ortiz, J., Pout, C.: A review on buildings energy consumption information. Energy and Buildings 40(3), 394 - 398 (2008). https://doi.org/10.1016/j.enbuild.2007.03.007

17. Saidur, R., Hasanuzzaman, M., Mahlia, T., Rahim, N., Mohammed, H.: Chillers energy consumption, energy savings and emission analysis in an institutional buildings. Energy 36(8), 5233 - 5238 (2011). https://doi.org/10.1016/j.energy.2011.06.027, pRES 2010

18. Wang, H.: Water flow rate models based on the pipe resistance and pressure difference in multiple parallel chiller systems. Energy and Buildings 75, 181 - 188 (2014). https://doi.org/10.1016/j.enbuild.2014.02.017

19. Zhao, X., Yang, M., Li, H.: Development, evaluation and validation of a robust virtual sensing method for determining water flow rate in chillers. HVAC\&R Research 18(5), 874-889 (10 2012). https://doi.org/10.1080/10789669.2012.667036 